\title{
Ricordi d'infanzia di Giosuè Carducci Tra prosa e poesia, tra realtà e mito
}

Matteo M. PEDRONI

Université de Lausanne

\begin{abstract}
Qua e là, nell'imponente opera letteraria e saggistica, Carducci ci parla della sua infanzia tra Versilia e Maremma toscana, attribuendo a brevi ricordi, a volte ricorrenti, funzioni che vanno ben al di là della mera cronaca autobiografica. Essi concorrono infatti alla costruzione del personaggio pubblico, del poeta come dell'intellettuale, e alla creazione di un mito identitario maremmano. Il presente articolo analizza alcuni di questi ricordi d'infanzia, iniziando da quelli in prosa e passando poi a quelli in versi.
\end{abstract}

Keywords: Carducci, ricordo, memoria, infanzia, mito.

A Gabriella Bertaccini e Uliano Trenta che mi hanno aperto le porte della Romagna quand'ero bambino

Ricevendo la cittadinanza onoraria di Bertinoro, che gli era stata conferita per l'impegno profuso, con opere e con parole - soprattutto con le parole «ornate ed alate» dell'ode famosa -, per riscattare dall'oblio e dalla rovina la Chiesa di Polenta, Carducci si rivolgeva alle autorità e ai cittadini con queste parole:

Io, componendo i versi su la Chiesa di Polenta, obbedii a un segreto mio genio, il quale, ovunque la terra italiana mostra le sue bellezze, ovunque la storia italiana parla con le sue memorie, mi comanda di accogliere quelle memorie, di rendere quelle bellezze con la parola ornata ed alata (Carducci 20I6: 27).

Sono parole che valgono per tutta quella poesia di Carducci che prende spunto dal paesaggio e dai monumenti per raccontare la storia italiana e così trasmettere ai contemporanei e alle generazioni future memorie e valori fondamentali. Memorie e valori che Carducci concepiva come fondamenti di una società civile, libera, giusta e sana.

Nella sua allocuzione il sindaco di Bertinoro precisava che la cornice del diploma di cittadino bertinorese, scritto in latino da Paolo Amaducci, era stata ricavata dal legno del cipresso descritto nei primissimi versi dell'ode:

I Discorso pronunciato davanti alla Chiesa di Polenta il Io settembre 20I6, in occasione del CI «Raduno carducciano» dell'Accademia dei Benigni di Bertinoro. Di quell'occasione si mantiene il tono e la forma conversevoli. 
Il cipresso che voi cantaste nell'ode alla Chiesa di Polenta l'arduo cipresso, che agile e solo veniva quasi accennando di colle in colle, fu nel pomeriggio del 2I luglio p. s. colpito ed atterrato da un fulmine. Ebbene il legno di questa cornice è tratto da quel ceppo! Si direbbe quasi che la natura per unirsi alle acclamazioni del popolo, abbia detto al cipresso: «Tu, che avesti il vanto di essere cantato dal maggior poeta, hai vissuto abbastanza! Colpito dal sacro fuoco del cielo, mori di morte gloriosa: spogliati, e cedi le tue spoglie per onorare il poeta che ti cantò!» (Carducci 20I6: 26-27).

Con la spiegazione dell'evento atmosferico in chiave «mitologica» il sindaco aveva, io credo, perso un po' la misura, e difatti Carducci nella risposta evitò di tornarci sopra. Ma quel che preme osservare è altro: è quel cipresso che - come glossano i commentatori - «viene incontro a chi sale di colle in colle [...] inchinando la chioma sotto il vento quasi in cenno di saluto»². Questo cipresso alto («arduo») e snello, («agile») schietto ricorda ben più noti cipressi «alti e schietti»; quelli che «balzano incontro» (v. 4) al Carducci che attraversa in treno il litorale maremmano nel I874; quelli che, riconoscendo il bambino che tante volte li aveva presi a sassate, gli chiedono «co 'l capo chino» (v. 6) di restare con loro. I cipressi che poi, una volta che il professor Carducci, avrà declinato con mesta autoironia il loro amichevole invito, agiteranno nuovamente il capo e lo compatiranno con un «ghigno pio» (v. 3I):

I cipressi che a Bólgheri alti e schietti

Van da San Guido in duplice filar,

Quasi in corsa giganti giovinetti

Mi balzarono incontro e mi guardâr (Davanti San Guido, vv. I-4).

E quando si congedano, $\mathrm{i}$ «cipressetti» non ricordano forse gli alberi del finale della Chiesa di Polenta? Nell'ode leggiamo: «mormoran gli alti vertici ONDEGGIANTI / Ave Maria» (vv. I27-I28); e in Davanti San Guido: «Un mormorio / Pe' dubiTANTI vertici ONDEGGIÒ» (vv. 29-30). Notate le ricorrenze lessicali: il «mormoran» dei cipressetti toscani ricorda il «mormorio» degli alberi bertinoresi; l'«ondeggianti», riecheggia l'«ondeggiò», e ancora notate «vertici» che riprende pari pari $\mathrm{i}$ «vertici» dei cipressetti.

Cipressi umanizzati, accoglienti, che per un'illusione ottica paiono venirci incontro e salutarci con un cenno della loro cima, che ci salutano con un rimbrotto o una preghiera. Questa analogia tra i due componimenti fa riflettere: pur piantati in luoghi lontani geograficamente, i primi in Toscana, il secondo in Romagna, e lontani testualmente, i primi nelle Rime nuove

2 «Agile e solo vien di colle in colle / quasi accennando l'ardüo cipresso» (Carducci, La chiesa di Polenta, vv. I-2, in Rime e Ritmi). 
il secondo in Rime e Ritmi, questi cipressi sembrano svolgere una medesima funzione, sottolineata dalla loro collocazione nella poesia: funzione - $\mathrm{di}$ rei - di soglia. Oltre quei cipressi c'è la memoria alla quale i cipressi stessi sembrano invitarci, venendoci incontro, premurosi, quasi a indicarci un luogo in cui - riprendendo le parole del poeta - «la terra italiana mostra le sue bellezze, [...] la storia italiana parla con le sue memorie». Il viaggio nello spazio, indicato dal movimento del treno e dall'ascesa al monte, diventa viaggio nel tempo, viaggio nella memoria. Alla fine di questo viaggio, l'albero ci risaluta, lasciandoci di nuovo nel presente, nella contemporaneità.

Memoria storica quella offerta dalla Chiesa di Polenta; memoria familiare quella di Davanti San Guido, nel rispetto di una bipartizione biografica su cui è tornato da ultimo Pantaleo Palmieri nell'introduzione all'importante fascicolo dedicato alla ristampa anastatica del primo tempestivo (1899) commento dell'ode carducciana, dovuto a Paolo Amaducci (Palmieri 20I6). Davvero una pubblicazione notevole, della cui iniziativa dobbiamo essere grati all'Accademia dei Benigni. Queste due poesie rappresentano emblematicamente quella bipartizione geografica, biografica e poetica, delimitata dall'Appennino, che Carducci varca nel I860 assumendo l'incarico di professore all'università di Bologna, e su quella cattedra resterà per più di quarant'anni, con una fedeltà che - a questi livelli di eccellenza scientifica e di notorietà internazionale - non ha paragone. Da quel momento non soltanto Carducci vivrà tra due "patrie» (chiamerà la Romagna «mia patria seconda» e Bologna «dilettissima seconda patria», OEN, IV, 223), ma assegnerà alla prima una valenza speciale, mitica.

Ciò che lo divide dalla Maremma non è soltanto l'Appennino ToscoEmiliano, ma una distanza temporale e spirituale; una Nostalgia, come recita il titolo di una rima nuova, di un'epoca conclusa che riemerge spesso nella memoria; vagheggiamento di una vita autentica, semplice e naturale, di cui la Maremma diventa simbolo. I luoghi dell'infanzia si mutano in un sentimento, in un "altrove" dell'anima, un rifugio dai crucci della vita, dagli obblighi e dalle fatiche professionali, dalla grettezza del presente, dalle delusioni poetiche e politiche. Il mito dell'infanzia toscana, che è perfettamente espresso in Davanti San Guido, sarà al centro di questo discorso: non parlerò però della fanciullezza di Carducci ma della sua rielaborazione attraverso la letteratura.

Le prose e le poesie in cui emergono i ricordi d'infanzia definiscono limpidamente la figura del vate, che attraverso la riflessione sulla storia, personale e nazionale, indica la strada da percorrere alla Terza Italia. I ricordi d'infanzia aprono prospettive illuminanti sul poeta, sul professore, sul 
patriota, sull'erudito, sul polemista, su quella complessa personalità che si affaccia anche nell'ode che ci riunisce qui oggi, davanti a quest'erma severa3.

Il ricordo d'infanzia, come strategia autobiografica, si apprezza anche nei versi dell'ultima stagione. Penso ai versi centrali di Piemonte, una delle sue odi celebrative più note, in cui - non a caso - Carducci innesta sul tema prettamente storico-politico, risorgimentale, quello dell'infanzia maremmana, dal quale - a ben vedere - è indissociabile, ed essenziale nella definizione del poeta nazionale.

Rievocando la prima guerra d'indipendenza, l'«anno de' portenti», il I848 e Carlo Alberto di Savoia, il Carducci cinquantacinquenne ricorda il Carducci tredicenne:

[...] Oh anno de' portenti, oh primavera de la patria, oh giorni, ultimi giorni del fiorente maggio, oh trionfante

suon de la prima italica vittoria che mi percosse il cuor fanciullo! Ond'io, vate d'Italia a la stagion più bella, in grige chiome

oggi ti canto, o re de' miei verd'anni (Piemonte, vv. 57-65).

La prima guerra d'indipendenza, con il fallimento dei moti rivoluzionari toscani, con la «fatal Novara», ma anche con le repubbliche mazziniane, segna la conclusione del periodo trascorso nella Maremma pisana - che oggi chiamiamo Maremma livornese o volterrana. «Quel tratto della Maremma - per intenderci e per dirla con le parole dello stesso Carducci - che va da Cecina a San Vincenzo» (LEN: XI, IO). In Maremma Carducci giunge all'età di tre anni: era nato nel luglio del I835 a Valdicastello, vicino a Pietrasanta, in Versilia, nell'entroterra tra Forte de' Marmi e Lido di Camaiore. Nel ' 38 la famigliola segue il padre, il dottor Michele, medico condotto, a Bolgheri, frazione di Castagneto; oggi Castagneto Carducci.

«Andare in maremma, allora - scrive ancora Carducci -, ci voleva coraggio: mio padre che v'andò con la moglie e due bambinelli, fu del tutto riputato pazzo» (LEN: XI, 9). La Maremma a quei tempi era zona selvaggia e insalubre e infatti Giosuè soffrì per due anni di «febbre maremmana», di febbri malariche, di cui si ricordò tanti anni dopo - nel I880 - nell'ode bar-

3 Il tradizionale raduno si svolge sul sagrato della chiesa di Polenta, proprio davanti all'erma di Carducci, opera dello scultore Garibaldo Alessandrini, inaugurata nel 1932. 
bara intitolata Una sera di San Pietro: «da poco avea scosso la febbre / maremmana, ed i nervi pesavanmi come di piombo» (vv. 6-7).

Michele Carducci era di idee liberali e carbonaro, temerario dunque se non veramente «pazzo» nella Toscana granducale. Per queste idee, dopo i moti rivoluzionari del '3I, a cui partecipò, aveva pagato caro, con il carcere e con un anno di confino a Volterra, dove comunque incontrò Ildegonda Celli, futura madre del poeta. A Michele Giosuè dovrà la sua prima educazione: l'apprendimento del latino e della metrica latina, la conoscenza di grandi scrittori, come Omero, che stimerà sempre come il più grande poeta: letto «con indicibile entusiasmo» da bambino e riletto altrettanto avidamente negli anni della maturità. In alcuni appunti autobiografici, risalenti al I878, c'è un'immagine suggestiva del giovanissimo Carducci che, «ai rossi crepuscoli della primavera maremmana», si protende dalla finestra a cercare la luce pur fievole che gli permetta di prolungare la lettura dell'Iliade. Sembra presagire il desiderio del vecchio poeta che, nella chiusa di Presso una Certosa, composta poco meno di un anno prima de La chiesa di Polenta, si augura che la poesia di Omero possa illuminare e confortare ancora la sua anima prima della morte:
A me, prima che l'inverno stringa l'anima mia
il tuo riso, o sacra luce, o divina poesia!
il tuo canto, o padre Omero,
pria che l'ombra avvolgami! (vv. I3-I6).

E noi sappiamo, da una testimonianza di uno degli ultimi suoi allievi, Manara Valgimigli, che «Carducci, nel penultimo anno di vita, si faceva [effettivamente] leggere i canti dell'Iliade» (Spaggiari 2007: 233). Nella «librerietta più che passabile per un medico di maremma» (LEN: XI, II), Giosuè ritrovava anche Dante, Petrarca, Tasso, e poi Berchet, Guerrazzi, Niccolini e Manzoni. Michele Carducci, come scrisse il figlio, era «mezzo-letterato, mezzo-poeta, mezzo-romantico e mezzo-rivoluzionario», ma «era un manzoniano fervente», non mezzo dunque ma intero, e quando c'era da punire il figlio non si peritava di picchiarlo, di rinchiuderlo, e soprattutto, quel che più addolorava l'intelligentissimo figlioletto, «voleva che io leggessi la Morale cattolica e l'Adelchi. E quindi cominciò il mio odio col Manzoni. Del quale prima avevo letto con molta curiosità per due volte i Promessi sposi» (LEN: XI, I2). Oltre alla letteratura, non pochi erano i libri di storia, «storia romana del Rollin, e la storia della rivoluzione francese del Thiers» (A proposito di alcuni giudizi su Alessandro Manzoni [I873], in Carducci I993: II, 400), che Giosuè leggeva con passione nelle lunghe giornate trascorse da solo, essendo il padre spesso lontano per il lavoro. 
«Grandi letture» che non si riducevano a un mero esercizio intellettuale, in cui la mente vibrava e il corpo si rammolliva: gli eroi della rivoluzione, i difensori della libertà repubblicana, antichi e moderni, spingevano al contrario il bambino all'azione, a tirar sassate non ai cipressetti, ma ai compagni di gioco. Leggiamo alcune righe in cui Carducci ci racconta appunto dei suoi giochi:

per ciò in brigata co' miei fratelli e con altri ragazzi del vicinato organizzavo sempre repubbliche, e repubbliche sempre nuove, ora rette ad arconti ora a consoli ora a tribuni, pur che la rivoluzione fosse la condizione normale dell'essere, e cosa di tutti i giorni l'urto tra i partiti e la guerra civile. La nostra repubblica consisteva di ragunanze tumultuose e di battaglie a colpi di sassi e bastoni, con le quali intendevamo riprodurre i più bei fatti de' tempi di Roma e della rivoluzione francese. [...] Che benedette sassate applicai un giorno a Cesare il quale era su 'l passare il Rubicone! per quel giorno il tiranno dové rifugiarsi non so dove con le sue legioni, e la repubblica fu salva (Carducci 1993: II, 4OI).

È una prosa che si fa apprezzare per lo spirito vivacissimo e lo stile brillante, che ci trascina dentro l'immaginario di quei fanciulli, che s'immedesimano in Giulio Cesare o Gaio Gracco o Publio Cornelio Scipione Emiliano o Lucio Opimio, con la sua scorta di guardie cretensi; o Luigi $\mathrm{XVI}$ con la sua scorta di guardie svizzere, massacrate dal popolo parigino nell'assalto alle Tuileries. Ma il gioco dura poco perché torna il dottor Michele e Giosuè-Gaio Gracco per le sue birbonate finisce in camera a leggere la Morale Cattolica del Manzoni, per poi però reincarnarsi nel tardo '400, in Guglielmo de' Pazzi che declama alla finestra alcuni versi di una tragedia alfieriana; ma sotto alla finestra, aggiunge Carducci, c'erano i suoi amici a deriderlo: «Dispetto! i cretensi e gli svizzeri eran sotto la finestra, e ridevano, e mi gittavano pomi» (Carducci 1993: II, 403).

A volte $\mathrm{i}$ «grandi fatti», le grandi azioni del piccolo Giosuè si concludevano - come abbiamo visto - con la prigionia e con le odiate letture manzoniane, ma altre volte egli riusciva a fuggire e se ne andava solo nella natura: «Quando mio padre mi sgridava un po" troppo, io fuggivo di casa e andava errando per le brughiere presso il mare e su le colline cretacee, e facevo lunghe meditazioni su le lucertole, i biacchi e i falchetti» (LEN: XI, I4). Si prefigura già in questa immagine quella particolare sfumatura caratteriale tra un Carducci virile, polemico, battagliero, che si azzuffa nella mischia o che alla mischia si rivolge sdegnoso, e un Carducci solitario, contemplativo, malinconico, ripiegato su di sé, sulle proprie sofferenze e insofferenze.

La Maremma, in quanto paesaggio contrastato, diventa il simbolo anche di questa complessa personalità. Basterebbe rileggere il celebre sonetto Traversando la maremma toscana, in cui, guardandola scorrere dal treno, Car- 
ducci, ormai cinquantenne, vede nel suo paesaggio l'origine e l'immagine della propria personalità: «Dolce paese, onde portai conforme / l'abito fiero e lo sdegnoso canto / e il petto ov'odio e amor mai non s'addorme» (vv. I-3). Un sonetto che si articola attorno alle frequenti antitesi («dolce» $v s$. «fiero e sdegnoso», «odio e amor», «tra il sorriso e il pianto»); antitesi che si sviluppano anche sul piano temporale: il passato dei sogni e delle speranze giovanili; il presente della delusione e della consapevolezza del fallimento di quei progetti e di quei sogni; e un futuro fatto di un' unica certezza, la morte. Eppure: «Pace dicono al cuor le tue colline / Con le nebbie sfumanti e il verde piano / Ridente ne le piogge mattutine» (vv. I2-I4).

Ovviamente non possiamo avvicinarci né alla prosa né tanto meno alla poesia autobiografiche come a fonti dirette della vita di un autore. Questo tipo di scrittura è parte integrante se non primaria nella costruzione dell'immagine pubblica di Carducci, in cui gli episodi sono, non dico inventati, ma selezionati e montati ad hoc per offrire una determinata immagine dell'uomo. Nel caso poi del racconto della propria infanzia, della quale nessuno è al corrente, e includo in quel nessuno anche il soggetto stesso che si racconta, che racconta a distanza di anni ciò che gli pare essere stata una realtà che invece in parte è frutto di una ricostruzione o rielaborazione memoriale (Zatti 2007: 303 ss.), il margine di adesione alla realtà dei fatti si assottiglia, ma non si assottiglia l'emozione che questa realtà ha provocato nel bambino e che si è mantenuta intatta nell'adulto. Il ricordo d'infanzia rappresenta la nascita della nostra coscienza attraverso la memoria e in un certo senso esso sostituisce il ricordo della nostra nascita biologica, di cui ovviamente non possiamo ricordare nulla. Studiare questi ricordi significa dunque confrontarci con un importante momento identitario per lo scrittore, che nel ricordo cerca sé stesso.

Degli anni versiliesi, dei primissimi dunque, Carducci ha voluto o ha potuto ricordare, in quell'occasione, soltanto due fatti:

Dei primi due anni e mezzo della mia vita, non ricordo se non la scoperta che io feci, con mia grande e seria meraviglia, di un bellissimo rospo nel giardino, mentre mi divertivo con una bambina; e la salita, che facevo con mia madre, le domeniche, di primavera e d'estate, alla Madonna di Stazzema (bellissima posizione), per la messa. Ricordo la salita al santuario: il di dentro del santuario, e la chiesa, nulla affatto (LEN: XI, IO).

Del primo ricordo, quello del rospo e della bambina, Carducci ha lasciato due versioni più ampie e particolareggiate. L'occasione per la stesura della seconda, risalente al I885, gli è porta da Emma Perodi, l'autrice delle Novelle della nonna, che allora dirigeva il «Giornale per i bambini»: la Perodi chiede a Carducci un suo ricordo d'infanzia da inserire in un libro pubblicato dallo stesso giornale. Il ricordo è breve ed è caro a Carducci, se poi lo ristamperà 
in apertura del suo volume di prose intitolato Confessioni e battaglie (1890). Vale la pena di leggerlo integralmente, prestando attenzione, prima di tutto, al modo in cui questo ricordo è raccontato: non tanto come un ricordo infantile, ma come un ricordo inappropriato alla lettura dei bambini e concesso controvoglia a una signora, Emma Perodi, alla quale per educazione non può essere rifiutato. Il ricordo di fatto è pensato per l'educazione dei lettori adulti e perciò nel libro dell' 85 non è inserito tra i ricordi d'infanzia degli altri uomini illustri, ma è anticipato nella prefazione della curatrice.

Si noterà poi il tono polemico e critico che innerva tutta questa pagina: polemica contro l'iniziativa della Perodi, che viene chiamata «figliuola d'Eva» e a cui si sarebbe dovuto dire di no; polemica - abituale in Carducci contro i manzoniani; polemica contro i costumi sociali in cui l'immoralità, travestita di «gravità» e di cultura, si arroga il diritto di giudicare: a un certo momento appare un «grave signore, con barba nera e con un libro in mano» che rimprovera i due bambini perché fanno troppo chiasso; quell'uomo - ci fa capire Carducci con un gustoso giro di parole - non è altro che l'amante di una donna sposata che abitava vicino a casa sua. Questo Ricordo dinfanzia è un autoritratto del Carducci adulto: difensore degli oppressi (anche degli animali) e della lingua italiana, fustigatore del malcostume, avversario della letteratura d'occasione; che porta avanti le sue battaglie provocando come in questo caso - gli avversari. Leggiamo il testo, che Giuseppe Chiarini, amico carissimo di Carducci, pose in apertura delle sue Memorie carducciane proprio per il suo carattere esemplare ${ }^{4}$ :

Mia signora,

Alle dimande delle figliuole d'Eva io credo che il più savio e sano partito sia sempre rispondere no. Questa volta, trattandosi di rimbambire per chiasso, non voglio parere villano.

Io della mia infanzia non ho memorie né belle né buone né curiose.

Il mio più antico ricordo mi pone súbito, ahimè, in relazione con un essere dell'altro sesso, come si direbbe con la lingua d'un certo uso, che, secondo i manzoniani, dovrebbe anche essere la lingua del buon gusto.

Mi ritrovo in un luogo né bello né brutto - forse un giardinetto presso la casa ove nacqui -, a una giornata, né di primavera né d'inverno, né d'estate né d'autunno. Mi pare che tutto, cielo e terra, sopra, sotto e d'intorno, fosse umido, grigio, basso, ristretto, indeterminato, penoso.

Io con una bambina dell'età mia, della quale non so chi sia o chi sia stata, dondolavamo, tenendola per i due capi, una fune; e mi pare che così dicevamo o credevamo di fare il serpente.

Quando, a un tratto, ci si scoperse tra i piedi una bella «bodda»: è il nome,

4 «Questo aneddoto mostra già nel fanciullo una delle qualità più caratteristiche dell'uomo, perciò l'ho messo qui, affinché sia come il battesimo della vita del nostro poeta» (Chiarini 1907: 6). 
nel dialetto della Versilia, d'un che di simile al rospo. Grandi ammirazioni ed esclamazioni di noi due creature nuove su quell'antica creatura.

Le esclamazioni pare fossero un po' rumorose. Perché un grave signore, con gran barba nera e con un libro in mano, si fece in sull'uscio a sgridarci, o meglio a sgridarmi. Non era mio Padre: era, seppi molto tempo dopo, un marito putativo d'una moglie altrui alloggiata per certo caso ivi presso.

Io, brandendo la fune, come fosse un flagello, me gli feci incontro, gridandogli: Via, via, brutto te!

D'allora in poi, ho risposto sempre così ad ogni autorità che sia venuta ad ammonirmi, con un libro in mano e un sottinteso in corpo, a nome della morale.

Ma veramente morale, per bambini, questa storia non è.

Che vuole che faccia io, signora? È storia. E ho ubbidito5 (Carducci 20oI: 27).

Converrete che questo è un modo assai particolare di raccontare la propria infanzia, privandola di quella poesia che pur Carducci - come abbiamo visto - vi sentiva, e puntando tutto sulla rappresentazione di sé come individuo scontroso e scomodo. Non solo Carducci non fa nessuno sforzo per abbellire il ricordo con dettagli verosimili anche se non appartenenti alla memoria, secondo una tendenza assai diffusa a romanzare; ma sottolinea il suo rifiuto di cedere a questo tipo di letteratura zuccherosa e sentimentale, negando le attese del pubblico con un accumulo di negazioni che eccede probabilmente i limiti della memoria: «Io della mia infanzia non ho memorie né belle né buone né curiose», «un luogo né bello né brutto», «una giornata né di primavera né d'inverno né d'estate né d'autunno», «una bambina dell'età mia, della quale non so chi sia o chi sia stata».

Sulla propria vita, e in particolare sulla propria infanzia, o Carducci decideva di scrivere di sua spontanea volontà, come fece nel ricordato saggio su Manzoni, oppure non c'erano santi che potessero smuoverlo. Su questo, come su molti altri fronti, era davvero irremovibile, a costo di diventare sgarbato oppure - come in questo caso - sottilmente odioso.

Era una reazione alla quantità di richieste dei suoi ammiratori, che gli chiedevano di giudicare i loro imparaticci poetici, che gli chiedevano di scrivere versi d'occasione, magari a pagamento, di pronunciare discorsi; che lo pregavano di partecipare ad avvenimenti culturali, a iniziative di beneficen$\mathrm{za}$, a "giornali politici o letterari, a riviste, a raccolte, a strenne, a numeri unici» $(O E N$ : XXV, 228). In più occasioni Carducci domanderà privatamente, per lettera, e pubblicamente, attraverso articoli di giornale, di essere lasciato in pace. Celebre è la lettera aperta indirizzata al direttore del «Resto del Carlino» del gennaio I887, in cui Carducci opponeva alle varie proposte di

5 Per alcune questioni filologiche legate al Ricordo d'infanzia e per la nascita della lettera ad Angelo De Gubernatis del I4 gennaio I878, più volte citata nel mio discorso (LEN: XI, 8-I4) rimando a Pedroni 2018. 
collaborazione un secco e reiterato non voglio e non posso. Si tratta di un vero e proprio Sfogo (OEN: XXV, 227-237), così s'intitola l'articolo, dallo stile inconfondibile com'è quello polemico di Carducci, in cui si esaltano a vicenda registri diversi: modi della lingua parlata e ricercatezze classiche, allusioni colte e toni gergali, apostrofi violente e metafore caricaturali. Carducci dice di no, perché è pagato per fare il professore e non lo scrivano; perché non crede nella letteratura d'intrattenimento, quella fatta per «divertire il genere umano»; perché l'Italia contemporanea produce troppa letteratura e di cattiva qualità: la definisce «un mercato di volgarizzazioni degli ultimi escrementi del romanticismo in prosa ed in verso»; perché è stufo di dover correggere «i soliti spropositi di morfologia e di sintassi che la teoria manzoniana ha fecondamente covato in tutte queste teste di scriccioli» (237):

Non voglio. Pe 'l rispetto, o, meglio, pe 'l concetto che ho dell'arte di dire e di scrivere e della libertà, io parlo e scrivo soltanto quando pare a me, cioè quando l'animo e l'intelletto me lo suggeriscono, e pubblico soltanto quando e come e dove credo opportuno. I signori i quali si compiacciono di chiedermi epigrafi e poesie mi onorano di reputarmi abile a far loro da segretario, per mettere in bello stile, stavo per dire in buona copia, i loro concetti o affetti. Di che io li ringrazio quanto so e posso grandemente, ma pur troppo sento che la natura mi ha costituito in modo da non poter far l'Annibal Caro né pagato né gratis. In somma, non scrivo per ordinazione o a richiesta. Quello di star seduto a sporcar di inchiostro la carta bianca e le dita nette, quando uno non ha da comunicare qualche cosa di nuovo e di buono, o almeno proprio, è un mestiere che io co' Romani abbandonerei agli schiavi (228).

L'avvertimento era dato, ma le richieste non cessarono e nell'epistolario non sono infrequenti i rifiuti perentori. L'eccezione fatta per la direttrice del «Giornale per i bambini» sembrava aprire comunque una crepa nella coriacea corazza del Maremmano, e c'è chi pensò bene, o male, d'insinuarcisi, per ottenere altri ricordi d'infanzia. Carducci non si scompose più di tanto, nemmeno di fronte alle richieste insistite da parte di un intellettuale, oggi del tut to dimenticato: Onorato Roux. Gli cedo volentieri la parola, perché mi sembra che il suo resoconto la dica lunga sulla coerenza di Carducci e anche sulla sua riservatezza quando si trattava di parlare della propria infanzia. Una riservatezza che è pari all'affetto per quell'infanzia maremmana che trova come privilegiato luogo di rimemorazione non la prosa (e certo non la prosa altrui, quella dei biografi), ma la poesia, come cercherò di mostrare nella seconda parte di questo discorso.

Onorato Roux raccolse in quattro volumi le memorie giovanili di illustri italiani, tra i quali non poteva mancare Giosuè Carducci, il maggior poeta dell'Italia unita. Ma nel libro di Roux si ristampa soltanto il ricordo del 
rospo e della bambina, perché Carducci non gliene spedì nessun altro. Di questo fatto Roux si giustifica nell'introduzione, che leggo:

Letto l'aneddoto della «bodda» narrato da Giosue Carducci in un libro intitolato: Ritorniamo piccini! pubblicato da Emma Perodi, allora direttrice del «Giornale dei bambini», e riprodotto poi nelle Opere del Poeta, mi procurai l'onore d'invitare il Carducci a scrivere i proprii ricordi infantili e giovanili. Ricevetti la seguente lettera:

Bologna. 6 nov. 89.

Mio signore,

Ma ci mancherebbe altro! Le pare?

Simil puerilità la feci una volta, per debolezza di non saper dir di no a una signora. Ma a Lei, maschio, posso ben dir di no.

Dev.

Giosuè Carducci

Insistetti; ed Enotrio Romano:

Bologna. 7 dec. 1889.

«Non bis in idem».

\section{Giosuè Carducci}

Ebbi, poi, più volte, occasione di avvicinare il Carducci, che, forse, in considerazione dello scopo altamente educativo del mio libro, mi promise, verbalmente, di farmi contento, aderendo finalmente all'invito; ma, siccome a me non giungevano le pagine autobiografiche promessemi, così, spiacendomi di non poter offrire alla Gioventù il nobile esempio della operosa vita giovanile del nostro maggior Poeta contemporaneo, feci un altro tentativo: gli riscrissi ed ebbi questa risposta:

Bologna, 24 marzo I902.

\section{Carissimo Signore,}

Mi rincresce che Ella perseveri nella sua illusione, che io Le abbia promesso di scrivere i miei ricordi d'infanzia e di giovinezza per gl'«Illustri Italiani contemporanei». Io non mi sono tenuto mai né così importante, né così esemplare, né così fatuo da scrivere le mie bambinate ad esempio. Ora poi che son vecchio, né mi posso più servir della mano per iscrivere, si figuri Lei s'io voglio sciupare il poco tempo che mi avanza e se voglio seccare gli altri a scrivere la mia epopea infantile. Ma Ella, caro signore, mi conosce poco davvero. 
Suo devotissimo
Giosuè Carducci.

Non mi detti per vinto. Replicai; ma, com'era da prevedersi, l'invitto Poeta mi rispose semplicemente così:

NO.

G. C.

Bologna. 20 maggio I905 (Roux I908: I6I-I63).

Non credo sia necessario commentare questa divertente sequenza di richieste e di rifiuti. Forse posso aggiungere che queste lettere Onorato Roux le ristampò su due giornali in occasione della morte di Carducci, per ricordarlo, per ricordarne il carattere fiero che ne fece un personaggio rispettato e anche temuto ${ }^{6}$.

Veniamo ora all'evocazione dell'infanzia maremmana nella poesia. Qui, più che per la prosa, vale la diffidenza da una considerazione puramente autobiografica dei contenuti memoriali. L'infanzia nei versi funziona spesso da reagente, da sfondo dal quale staccare e contrapporre la condizione esistenziale dell'io poetico adulto. Non è mai dunque un ricordo che vale per sé, ma sempre si giustifica per il suo valore emblematico ed universale. Senza contare l'estrema formalizzazione della poesia rispetto alla prosa: l'espressione poetica coinvolge una serie di elementi senza i quali, soprattutto per un classicista come Carducci, non vi può essere poesia. Il ricordo infantile deve perciò armonizzarsi con l'elaborazione retorica, con le scelte metriche, con la memoria letteraria, che lo pone in dialogo con altri testi, propri e altrui. Nell'opera poetica di Carducci, nella sua configurazione definitiva e più nota, quella cioè delle raccolte canoniche, organizzate dall'autore a partire dalla fine degli anni '8o - Juvenilia, Levia Gravia, Giambi ed Epodi, Rime nuove, Odi barbare e Rime e Ritmi -, la memoria dell'infanzia maremmana si concentra soprattutto nel quinto libro delle Rime nuove: vi ritroviamo infatti Rimembranze di scuola, Idillio maremmano e Davanti San Guido. Non che in altri componimenti non si possano rintracciare allusioni agli anni della fanciullezza e della prima adolescenza, tra Bolgheri e Castagneto, ma in nessuno - tranne in Sogno d'estate - la memoria infantile assume una

6 Le lettere, non so se già in questa forma, vennero pubblicate sul Giornale d'Italia, Roma, 19 febbraio 1907 e sul Resto del Carlino, Bologna, 19-20 febbraio 1907. 
forma narrativa così compiuta, in nessuno lo sforzo di ricostruire una realtà determinata e concreta, storica, è così spinto.

La Maremma è il luogo e il tempo delle prime indimenticabili esperienze, quelle che hanno segnato la vita di noi tutti e che - come scrisse Carducci - «percuotono il cuor fanciullo»; sono le esperienze di cui Sigmund Freud capirà l'importanza per la formazione dell'adulto. Secondo Freud

alcune esperienze precoci si fissano nella nostra mente e ci impongono un certo modo di desiderare, di amare, e in definitiva di vivere. Perciò esse danno un senso alle nostre vite. [...] Per Freud la storia di una vita è essenzialmente la storia di un desiderio così come esso si è definito a partire dalle esperienze infantili (Brugnolo 20I2: II).

Per Carducci la Maremma significa anche la primissima esperienza poetica, ispirazione e scrittura. In alcuni appunti autobiografici, pubblicati soltanto postumi, un Carducci poco più che adolescente dedica alcune pagine proprio a elencare i suoi Primi componimenti (OEN: XXX, 6-9). Si tratta di un elenco di titoli e di contenuti di composizioni nate attorno a letture o a esercitazioni scolastiche. Soltanto in un caso l'ispirazione è attribuita alla contemplazione del paesaggio infantile e non allo studio libresco; a un impulso che muove dal cuore e non dall'intelletto:

Passato con la famiglia a Castagneto fui messo a scuola da un prete [...] e io chiesi a questo prete che mi facesse comporre. [...] A questo metodico maestro chiesi di far poesia; non me lo assentì. Ma io o anteriormente o posteriormente o contemporaneamente (che più non mi ricordo) non già a tavolino e a furia di cassature, ma mentre da una finestra di casa mia [ammiravo] una di quelle care sere di Maggio che tanto parlano al cuore dell'adolescenza; e inspirato dalla campana che suonava la prima ora di notte, avea scritto il mio primo sonetto (8).

Di questa prima esperienza restano tracce anche nella poesia, ma non nella forma della narrazione di un episodio determinato, piuttosto in quella dell'allusione, in cui i dettagli realistici vengono meno e il senso si condensa in topoi personali e ricorrenti, come quello dei colli toscani: «Né vi riveggo mai, toscani colli, / Colli toscani ove il mio canto nacque» (Per Val d'Arno, Levia Gravia, vv. I-2); o ancora: «Là in fondo sono $i$ miei colli, / con la serena vista, con le memorie pie. // Ivi m'arrise fanciullo la diva sembianza d'Omero» (Pe 'l Chiarone da Civitavecchia, Odi Barbare, vv. 57-59), e anche in un contesto comico-satirico, quello di Intermezzo: «io, ripigliato sonno, ancora voi, / miei colli, rivedeva, / miei dolci colli, ove tra' lauri move / l'arte serena l'orme» (vv. 95-98). 
Ecco che la visione del paesaggio dell'infanzia, concentrato nell'unica immagine dei suoi «colli», si unisce alla nascita della vocazione poetica e si trasfigura attraverso la reminiscenza foscoliana («Né mai più toccherò le sacre sponde / ove il mio corpo fanciulletto giacque»), l'evocazione omerica e, in tutte, attraverso l'elaborazione retorica (anadiplosi, chiasmo, allitterazione, ecc.) in un'esperienza che non ha più nulla della cronaca autobiografica.

Diverso è invece il caso di quelle poesie raccolte nel quinto libro delle Rime nuove, tra le più celebri del Carducci scolastico, almeno fino a qualche anno fa. In questi «idilli» (Martini 2005) la fanciullezza è restituita nella concretezza di un episodio o anche solo di un gesto compiuto o di un sentimento provato dal Carducci bambino, che vi può apparire anche come personaggio.

Cosa dicono i cipressi a Carducci in Davanti San Guido?: «ira non ti serbiam de le sassate / tue d'una volta». Tirare sassate contro gli alberi o contro - cosa ben più grave - altre persone (ricordate le «benedette sassate applicate un giorno a Cesare»), è un gesto comune a tantissimi bambini, di ogni luogo e di ogni tempo, ma è pur sempre un gesto del piccolo Giosuè Carducci. Un gesto non certo ricordato a caso, né nella prosa autobiografica né in Davanti San Guido, basti leggere qualche verso più avanti, la risposta del Carducci adulto ai cipressetti: «non son più, cipressetti, un birichino, e sassi in specie non ne tirò più. // E massime a le piante» (vv. 27-29). Le sassate del bambino contro gli alberi e poi contro i giocosi nemici delle repubbliche diventano le metaforiche sassate del Carducci adulto, che si batte per i propri valori, scagliandosi pubblicamente contro i nemici dell'Italia. Quante polemiche e dibattiti sollevò Carducci durante la sua vita, quanti «Via, via, brutto, te!» pronunciò contro la vigliaccheria, la ciarlataneria, l'istrioneria del suo tempo?

E non è forse dai «toscani colli», dal paesaggio dell'infanzia, che Carducci eredita questo carattere, come rivela il già ricordato sonetto Traversando la maremma toscana?: «Dolce paese, onde portai conforme / l'abito fiero e lo sdegnoso canto / e il petto ov'odio e amor mai non s'addorme» (vv. I-3). Come sottolinea Marco Veglia, il paesaggio carducciano è paesaggio etico prima ancora che estetico (Veglia 2007). Nel paesaggio - in cui vive, nel presente, la storia dei popoli che l'hanno plasmato - Carducci legge una regola di vita. Nel paesaggio maremmano è iscritto il destino di Carducci, perché nascendo in quel luogo, che porta in sé il carattere dei popoli che l'hanno abitato e modificato, Carducci non può che ereditarne i valori. L'etica che la Maremma toscana comunica è quella stessa a cui i cipressetti richiamano un

7 «Io credo di dover mettere al nudo la vigliaccheria la ciarlataneria la istrioneria che guasta e macera la nazione. Se altri facesse in politica quello che io in letteratura, l'Italia non sarebbe quello che è, o almeno non sarebbe per un pezzo» (LEN: XIII, I28, a don Giovanni Battista e A Prato, 9 giugno 188I). 
Carducci che se ha conservato intatto «l'abito fiero e lo sdegnoso canto», ha però dimenticato il loro pendant, il loro complemento naturale, la serenità ${ }^{8}$.

Intesi allora che i cipressi e il sole
Una gentile pietade avean di me,
E presto il mormorio si fe' parole:
- ben lo sappiamo: un pover uom tu se'.
Ben lo sappiamo, e il vento ce lo disse
Che rapisce degli uomini il sospir,
Come dentro al tuo petto eterne risse
Ardon che tu né sai né puoi lenir.
A le querce ed a noi qui puoi contare
L'umana tua tristezza e il vostro duol.
Vedi come pacato e azzurro è il mare,
Come ridente a lui discende il sol! (vv.33-44).

I cipressetti gliela vorrebbero offrire (vv. 53-64) la serenità, purché si fer$\mathrm{mi}$ in mezzo a loro; gliela promettono ${ }^{9}$, ma Carducci deve andare e i cipressi, come ultima risorsa, prima di congedarsi «brontolando», gli ricordano la nonna Lucia in cimitero: "Che vuoi che diciam dunque al cimitero / dove la nonna tua sepolta sta?» (vv. 73-74). A questo punto ecco apparire la nonna Lucia, nel secondo ricordo veramente personale e concreto dell'infanzia, dopo quello delle sassate: la nonna che racconta al nipote «la novella di [chi] cerca il suo perduto amor» e che di fatto anticipava il triste destino del Carducci. I cipressi sanno che nella nonna, e in nessun altro, il piccolo Giosuè trovava rifugio dalle avversità, sulle sue ginocchia, ascoltando le dolci novelle. Così la ricorda Carducci, sulla soglia dei quarant'anni, in una lettera del I874, diretta all'amante, Carolina Cristofori Piva:

Il mio primo dolore, del quale serbo vivissima rimembranza, fu la morte della mia nonna paterna. [...] io mi ricordo bene che il mio cuore infantile ne fu straziato, mi ricordo bene che allora intesi orribilmente che fosse la morte: quella donna, che mi teneva su le ginocchia, che mi raccontava le lunghe e belle fantastiche novelle, che mi dava ragione e mi consolava delle ingiurie materne e delle paterne crudeltà andava a giacere sotterra, nel povero camposanto, nello stridore del verno $(E N L \text { : IX, 2I } 8)^{\mathrm{IO}}$.

8 Con la serenità, la pace dell'anima si concludeva anche la contemplazione del paesaggio nel già citato sonetto Traversando la maremma toscana: «Ma di lontano / pace dicono al cuore le tue colline» (vv. II-I2).

9 «Il dissidio, o mortal, de le tue cure / Ne la diva armonia sommergerà» (vv. 63-64).

Io Si veda pure Di notte, in Rime nuove, vv. I2-I4: «ma posa trovo in te, qual pargoletto / che singhiozza e s'addorme de la pia / ava abbrunata su l'antico petto». 
Il ricordo d'infanzia si configura come un destino, in cui l'adulto si riconosce, in cui trova un senso alla propria esistenza e una ragione alla propria individualità. Tutti i ricordi d'infanzia di Carducci sono fatali, esperienze spesso traumatiche, che mutano per sempre la vita dell'individuo, escludendolo dal paradiso dell'infanzia, dalla spensieratezza, e indicandogli le prove future. La spensieratezza infantile stava appunto nell'assenza di ricordi e dunque di dolori, dolori e ricordi che Carducci riconnette spesso alla sua condizione adulta, come a sottolineare il carattere profetico di quelle esperienze: «Da allora in poi...» (Ricordo dinfanzia), «Ond'io [...] oggi ti canto, o re de' miei verd'anni» (Piemonte), «Anche oggi» nel saggio su Goffredo Mameli (I872), in cui la commozione provata dal «fanciullo» sulle parole dell'Inno famoso prelude alla fede politica dell'autore dei Giambi ed Epodi:

Io ero ancora fanciullo; ma queste magiche parole, anche senza la musica, mi mettevano i brividi per tutte le ossa; e anche oggi ripetendole mi si inumidiscono gli occhi. Se non che oggi l'età è scettica e positiva; e a più d'uno darà per avventura molestia quell'«elmo di Scipio», mito da panche di scuola. [...] La sarà, se volete, retorica; perché oggi certa gente chiama retorica tutto quello che ha il torto di parlare al cuore e alla mente dei buoni e gentili un po' più presto e un po' più efficacemente che non le loro cifre e i resoconti, le quali e i quali han poi bisogno d'una retorica tutta speciale per apparire quello che non sono: ora io tra retorica e retorica scelgo la più bella ed onesta (Goffredo Mameli, in Carducci 1993: 365, il corsivo è mio).

Questa strategia autobiografica si riconosce anche in Rimembranze di scuola (I87I), che fa da pendant poetico alla prosa epistolare ricordata sopra e che lega la scomparsa della nonna paterna alla prima straziante rivelazione della morte: «Ancoggi / Quel fanciullesco imaginar risale / Ne la memoria mia» (vv. 48-5I, il corsivo è mio).

Si tratta della poesia in cui Carducci si rivede bambino nel modo più disteso, più naturale. A ciò molto contribuisce una forma metrica anomala per il Carducci delle Rime nuove come l'endecasillabo sciolto, immediatamente riconducibile al Leopardi delle Ricordanze, modello inimitabile di quella poesia della memoria che in quel periodo Carducci meditava profondamente.

In una giornata calda di giugno il piccolo Giosuè si annoia a scuola ascoltando il prete che coniuga «con voce chioccia» il verbo amare. Si annoia probabilmente un po' perché il suo livello di latino, grazie all'educazione che gli impartisce il padre, è molto avanzato rispetto a quello dei suoi compagni; e un po' perché l'amare di quell'arido insegnamento è incompatibile con lo spettacolo che si offre oltre la finestra. Fuori la natura è un'esplosione di vitalità e d'amore, non dell'amore biascicato, «bestemmiato» dal «nero prete», ma l'amore intenso della Natura infuocata: gli alberi centenari 
sembrano parlare con gli uccellini, che cantano felici, le farfalle baciano i fiori, tutto «formicolava d'indistinti amori»:
Non rideva io fanciullo: il nero prete
Con voce chioccia bestemmiava Io amo,
Ed un fastidio era il suo viso: intanto
A la finestra de la scuola ardito
S'affacciava un ciliegio, e co' vermigli
Frutti allegro ammiccava e arcane storie
Bisbigliava con l'aura. Onde, oblïato
Il prete e delle coniugazïoni
In su la gialla pagina le file
Quai di formiche ne la creta grigia,
Io tutto desïoso liberava
Gli occhi e il pensier per la finestra (vv. 7-18).

Lo spettacolo della natura rinata a gioventù dopo il periodo invernale, fa scattare nel bimbo un sentimento inaudito e straziante: l'«intendimento» della morte individuale, del non essere più, a cui si sovrappone il giudizio dei vivi, nel pensare la condizione buia, fredda, muta del cadavere:

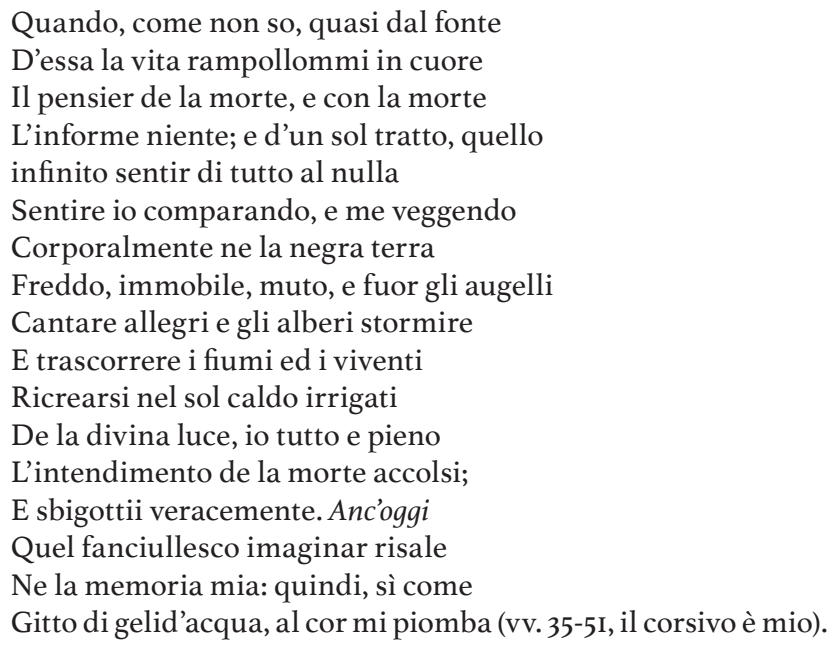

Il ricordo di sé bambino non può essere separato dal ricordo del proprio bambino, morto un anno prima della stesura di questo idillio, nel novembre del I870, a sua volta strettamente connesso alla celeberrima anacreontica Pianto antico: «L'albero a cui tendevi la pargoletta mano...». Anche in questo testo ritroviamo il contrasto tra l'eternità della Natura e la finitezza del destino individuale; la «negra terra» in cui si proiettava il piccolo Giosuè 
(Rimembranze di scuola, v. 4I) e in cui ora è sepolto il suo amatissimo figlio («Sei nella terra negra», Pianto antico, v. I4), «l'estremo unico fior».

Abbiamo finora considerato l'origine del carattere fiero e combattivo, che Carducci fa risalire da una parte al paesaggio di Traversando la maremma toscana, dall'altra alla difesa di una bambina nel Ricordo dinfanzia; abbiamo poi individuato il «primo intendimento de la morte», provocato dallo spettacolo della natura primaverile nelle Rimembranze di scuola e dalla morte della nonna Lucia nella lettera a Lina. Veniamo ora, per concludere, alla terza fondamentale rivelazione infantile, quella dell'amore.

Anche in questo caso il recupero memoriale si piega alle esigenze della scrittura, alle strategie autobiografiche che Carducci mette in atto specialmente in poesia, lasciando invece che nella prosa di memoria, solitamente privata, la realtà storica prevalga sulla costruzione simbolica.

Nell'Idillio maremmano (1873) Carducci rimpiange l'«amor mio primo, o d'amor dolce aurora» (v. 6). Un raggio di sole primaverile entra nella stanza del poeta e risveglia in lui l'immagine florida della bellissima «Maria bionda» (v. 3), amata nella Maremma della sua giovinezza. La concretezza del ricordo in questo caso consiste nella descrizione fisica della ragazza, sensuale, quasi provocante. Ma Maria era la promessa di una vita, per l'appunto, concreta, pura e sana; una vita rustica, semplice e serena. «Meglio era sposar te, bionda Maria! / [...] Che sudar dietro al piccioletto verso!» (vv. 33/37): dal ricordo nasce il rimpianto e dal rimpianto la considerazione della propria vita presente, triste e vana. Maria è l'incarnazione del paesaggio maremmano, ne è il frutto naturale; è la garanzia del nesso inestricabile tra Storia e Natura. In questo senso la figura femminile fa parte del paesaggio etico di Carducci, come i cipressetti, che non avrebbe mai dovuto lasciare. Ancora una volta il ricordo d'infanzia mette Carducci di fronte all'impossibilità di tornare non tanto in un luogo ma in una condizione esistenziale che gli era stata destinata:

Oh come fredda indi la vita mia, come scura e incresciosa è trapassata!

Meglio era sposar te, bionda Maria! (vv.3I-33)

Agli sforzi di identificare Maria con qualche ragazza amata dal poeta giovinetto, bisogna opporre le ragioni poetiche che ne fanno un simbolo: di fronte a certo positivismo biografico meglio credere al vecchio poeta quando scriveva al direttore del «Resto del Carlino» che l'Idillio maremmano «non ha parola che rassomigli a verità» (Carducci 1965: 26I).

Per rintracciare il primo vero amore di Carducci ci si dovrà rifare alla prima stesura di quel ricordo del rospo e della bambina di cui si è già detto, e che si legge nei Ricordi della mia vita fino a 15 anni, in cui l'adolescente ferma 
sulla carta episodi della propria infanzia per uno scrupolo di conservazione memoriale decisamente privato. Nel secondo capitolo, intitolato Il mio paese, si ritrova la scena che trentacinque anni dopo Carducci concederà controvoglia a Emma Perodi. Ma se nel ricordo offerto agli italiani, agli adulti e non ai bambini, primeggia lo «sdegno» del piccolo Giosuè di fronte all'autorità e al malcostume, nel diario del quindicenne - che si sforza di ricostruire non solo le azioni ma anche i sentimenti che le hanno motivate - questo stesso «sdegno» nasce dall'«amore». Bisognerà partire dai rospi, dalle dialettali «botte», che nella versione privata non hanno ancora la centralità che assumeranno in quella pubblica:

Mi ricordo della bella casina in cui si stava e del giardinetto annesso, e delle botte che la sera ci andavano a processione; e che per quel giardinetto trascinavano sopra un carriuccio me e mio fratello, bambinone spesse volte berciante. Mi ricordo, vivamente, di una bambina con cui facevo il chiasso. $\mathrm{E}$ fra le altre mi ricordo che una sera tarduccio ambedue ci trastullavamo con una fune o meglio (secondo il modo del mio paese) alla biscia che gettavamo in qua e là ad altalena, gridando al serpe al serpe al serpe, quando venne un uomo avanzato d'età che ci sgridò e recò via la bambina; e mi ricordo che io ne provai vivo dolore. Io ora penso fra me: quale furono le cose che influirono si prepotentemente su questo fatterello tanto inconcludente della mia fanciullezza perché io me ne ricordi con sì gran viva verità? E riandando alcuni altri miei fatterelli che mi son rimasti impressi per cause o di affetto che ho sentito o di sdegno che ho provato, ne induco che sulla viva ricordanza che ho sempre avuto di cotesta scena influissero e l'amore che io avevo a la bambina e lo sdegno che quell'uomo sgridasse ambedue e recasse via lei. E forse l'animo mio potentissimo ora nell'amore e nello sdegno, era tale anche a tre anni. [...] per cui oso dire che a 3 anni io amassi quella bambina d'amore (OEN: XXX, 5-6).

Si tratta di un documento notevolissimo per capire il rapporto esistente tra il ricordo della fanciullezza, la sua interpretazione in pectore da parte dell'io e la scrittura letteraria, che di quell'io deve veicolare un'immagine definita, pur non sempre fedele ai fatti, a quelli che ciascuno di noi crede siano realmente avvenuti un tempo lontano.

L'erma accigliata di Carducci invita a concludere.

\section{Bibliografia}

Brugnolo, Stefano, «Alcuni influssi freudiani sul tema letterario del ricordo infantile», Il ricordo d'infanzia nelle letterature del Novecento, a cura di Brugnolo S., Pisa, Facini, 20I2, pp. II-46. 
Carducci, Giosuè, Opere (OEN), Edizione Nazionale, Bologna, Zanichelli, I935-1940.

-. Lettere (LEN), Edizione Nazionale, Bologna, Zanichelli, 1938-1968.

-. Rime nuove, Testimonianze, interpretazione, commento di P.P. Trompeo e G. Salinari, Bologna, Zanichelli, 1965.

-. Opere scelte, a cura di M. Saccenti, Torino, Utet, 1993.

-. Poesie, a cura di W. Spaggiari, Milano, Feltrinelli, 2007.

-. Confessioni e battaglie, Edizione Nazionale, a cura di M. Saccenti, Modena, Mucchi, 200I.

- La Chiesa di Polenta, Ristampa anastatica dell'Ode e del Commento di P. Amaducci, a cura dell'Accademia dei Benigni di Bertinoro, [a cura di Milvia Del Zozzo e Fabrizio Fornasari], con un saggio di P. Palmieri, Bertinoro, GE.GRAF, 20I6, pp. 9-35.

Chiarini, Giuseppe, Memorie della vita di Giosue Carducci (I835-1907), Raccolte da un amico (Giuseppe Chiarini), seconda edizione corretta e accresciuta, Firenze, Barbèra, 1907.

Martini, Alessandro, «Le "Rime nuove" di Giosue Carducci e gli idilli del quinto libro", Architektur der Wolken. Zyklisierung in der europäischen Lyrik des 19. Jahrhunderts, a cura di R. Fieguth e Martini, A., Bern, Peter Lang, 2005, pp. 328-343.

Palmieri, Pantaleo, «Là dove torva l'aquila del vecchio Guido covava», in Carducci 20I6, pp. 9-35.

Pedroni, Matteo M., «"Io non voglio polemizzare co 'l Prof. De Gubernatis”. Logiche del malinteso in un carteggio carducciano», Carducci prosatore, Convegno organizzato dall'Università Statale di Milano, Gargnano del Garda, 29-30 settembre 20I6, in corso di stampa.

Roux, Onorato, Illustri italiani contemporanei. Memorie giovanili autobiografiche di letterati, artisti, scienziati, uomini politici, patrioti e pubblicisti, raccolte e corredate di cenni biografici, Edizione popolare, volume I - Letterati, Parte prima, Firenze, Bemporad, 1908.

Veglia, Marco, «Paesaggi carducciani», Carducci e i miti della bellezza, a cura di M.A. Bazzocchi e S. Santucci, Bologna, Bononia University Press, 2007, pp. I2O-I27.

Zatti, Sergio, «Raccontare la propria infanzia», in Francesco Orlando, Infanzia, memoria e storia da Rousseau ai Romantici, Pisa, Pacini, 2007, pp. 275329. 

